\title{
Scattering of electromagnetic waves by two crossing metallic single-walled carbon nanotubes of finite length
}

\author{
A. V. Melnikov' ${ }^{1}$, P. P. Kuzhir ${ }^{2,1}$, S. A. Maksimenko ${ }^{1,3}$, G. Y. \\ Slepyan $^{4}$, A. Boag 4 , O. Pulci ${ }^{5,6}$, I. A. Shelykh ${ }^{7,8}$, M. V. Shuba ${ }^{1,3}$ \\ ${ }^{1}$ Institute for Nuclear Problems, Belarusian State University, Bobruiskaya 11, 220050 Minsk, Belarus \\ ${ }^{2}$ Institute of Photonics, University of Eastern Finland, Yliopistokatu 7, FI-80101 Joensuu, Finland \\ ${ }^{3}$ Tomsk State University, Lenin Avenue 36, 634050, Tomsk, Russia \\ ${ }^{4}$ School of Electrical Engineering, Tel Aviv University, Tel Aviv 69978, Israel \\ ${ }^{5}$ Dept. of Physics, University of Rome Tor Vergata, \\ and INFN, Via della Ricerca Scientifica 1, I-00133, Rome, Italy \\ ${ }^{6}$ CNR-ISM, Via del Fosso del Cavaliere 100, 00133 Rome, Italy \\ ${ }^{7}$ ITMO University, Saint Petersburg, 197101, Russia and \\ ${ }^{8}$ Science Institute, University of Iceland, Dunhagi 3, IS-10\%, Reykjavik, Iceland
}

\begin{abstract}
The scattering theory for two crossing finite-length metallic single-walled carbon nanotubes exposed to an electromagnetic field has been developed based on a synthesis of the quantum transport formalism and classical electrodynamics. The model of the point contact has been developed to be incorporated into Hallén and Pocklington equations for crossing carbon nanotubes. The influence of the contact conductance and position as well as angle between the tube axes on the tube polarizability has been analyzed in the range of $1 \mathrm{GHz}-10 \mathrm{THz}$. The physical mechanisms responsible for the electromagnetic interaction between crossing tubes with zero and non-zero intertube contact conductance are shown and discussed. The influence of the coupling between the tubes on the localized plasmon resonance in them has been demonstrated.
\end{abstract}

PACS numbers: 42.70.-a, 73.25.+i, 77.84.Lf, 78.67.Ch

\section{INTRODUCTION}

Electromagnetic properties of both individual carbon nanotubes (CNTs) and CNT-networks have been actively studied during the past two decades ${ }^{1-8}$. The theory of electromagnetic (EM) wave scattering has been developed for individual single-walled CNT $(\mathrm{SWCNT})^{2-4}$, SWCNT bundle ${ }^{9}$, multiwalled $\mathrm{CNT}^{10}$, curved $\mathrm{CNT}^{11}$, SWCNT with mesoscopic insertion ${ }^{8}$, and SWCNT with dielectric coating ${ }^{12}$. Many interesting electromagnetic phenomena have been predicted and discovered in CNTs including slowed-down surface waves ${ }^{1}$, antenna effect in finite-length $\mathrm{CNTs}^{2-4}$, screening effect due to the strong depolarizing field ${ }^{12}$, near-field enhancement ${ }^{13}$, Purcell effect $^{14}$, and specific thermal radiation ${ }^{15}$. The first antenna resonance or, in other words, localised plasmon resonance (LPR) in CNTs has been observed as a broad peak in the conductivity spectra of CNT films ${ }^{16}$. Peak frequency has been shown to vary from 3 to $10 \mathrm{THz}$ when the tube length decreases from $1 \mu \mathrm{m}$ to $300 \mathrm{~nm}^{6}$. Moreover, the magnitude of the terahertz conductivity of CNT films strongly depends on the CNT length demonstrating relatively weak coupling between adjacent CNTs in their conductive network in the terahertz range ${ }^{17}$.

Though the electromagnetic response has been reported for periodic CNT array ${ }^{18-20}$ and bundles of $\mathrm{CNTs}^{9}$, the in-depth analysis of the interaction between even two non-parallel CNTs has not been done yet. Also, the electromagnetic theory of CNT network taking into account both electromagnetic interaction and intertube transport between adjacent CNTs has not been developed yet. Note, that modeling of the coupling at the nanoscale is quite complicated as it should take into account both the electromagnetic coupling and overlapping of the wave functions ${ }^{21-23}$. Currently, only the mutual impedance of two non-touching CNT antennas has been studied $^{2,24}$.

Recently, simple Waterman-Truell approach has been successfully applied in Ref. 25 to describe conductivity spectrum of CNT film in the terahertz range. The main approximation of this approach is the omission of the intertube interaction. Justification of this simplification has not been given yet due to a high complexity of the problem. Let us point out the inconsistencies between the experimental data and the theoretical description based on the Waterman-Truell approach in the frequency range below the LPR peak: (i) frequency dependence of the measured effective conductivity of the CNT film is weaker than that predicted by the theory ${ }^{26}$; (ii) concentration dependence of the effective conductivity follows power-law behavior with the exponent being larger than unity $^{27,28}$, whereas the Waterman-Truell approach predicts linear dependence; (iii) dielectric loss tangent of CNT film appears to be much higher than that obtained from the theory ${ }^{17}$. We believe that introduction of the intertube electron transport into the model of electromagnetic interaction between the CNTs can reduce inconsistencies between the experimental data and the theoretical description.

Here, we present a scattering theory for two interacting SWCNTs as a first step toward developing the theory of CNT-networks. We formulate an electrodynamic boundary-value problem for two crossing finite-length carbon nanotubes exposed to an electromagnetic field. 
The problem is reduced to both Hallén and Pocklington equations. The conductance of the contact between CNTs is described by the quantum transport theory of the mesoscopic structure. The intertube current is taken into account by (i) a discontinuity of axial currents in CNTs, and (ii) by an introduction of the extra charges at the crossing points of the CNTs. The polarizabilities of crossing CNTs are calculated and analyzed. The influence of the tunneling and electromagnetic coupling between the adjacent tubes on the localized plasmon resonance in the CNTs is shown. Our study provides a justification of the applicability of Waterman-Truell approach for CNT network at and above the frequency of the LPR. On the other hand, we demonstrate that the intertube coupling cannot be excluded from consideration in the frequency range below the LPR. This paper is a continuation of our research on the synthesis of the electrodynamics and the theory of electrical nano-circuits ${ }^{8}$.

\section{THEORETICAL FRAMEWORK}

\section{A. Boundary-value problem}

Let a system of two crossing finite-length single-wall carbon nanotubes be located in a host medium with permittivity $\varepsilon$ (host relative permittivity is $\varepsilon_{h}=\varepsilon / \varepsilon_{0}$, $\varepsilon_{0}=8.85 \times 10^{-12} \mathrm{~F} / \mathrm{m}$ ) (see Fig. 1). Let us designate the CNTs by index $m, m=1,2$. The $m$-th tube is of radius $a_{m}$, length $L_{m}$, and has axial surface conductivity $\sigma_{m}$. The angle between the CNT axes is $\theta(\theta \leq \pi / 2)$, and the shortest distance between them is $D+a_{1}+a_{2}$, where $D$ is a distance between the surfaces of the tubes at the crossing point $(D \geq 0.25 \mathrm{~nm}$, see Ref. 29$)$.

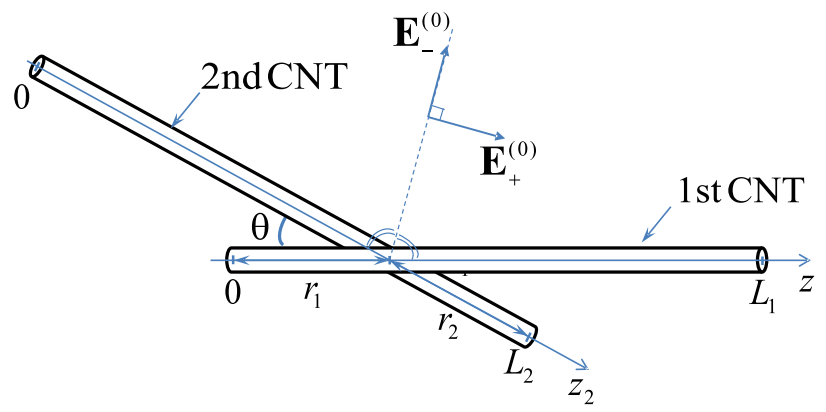

FIG. 1. Geometry of two crossing CNTs of lengths $L_{1}$ and $L_{2}$. Axes $z$ and $z_{2}$ coincide with the axes of the 1st and 2nd CNTs, respectively. Also, $r_{1}$ and $r_{2}$ denote the distances between CNT edges and the crossing point. Vectors $\mathbf{E}_{ \pm}^{(0)}$ show the direction of the incident fields taken for numerical calculations in Sec. III.

Let us choose two cylindrical coordinate systems $(\rho, \phi, z)$ and $\left(\rho_{2}, \phi_{2}, z_{2}\right)$ so that the axes $z$ and $z_{2}$ coincide with the axes of the 1st and 2nd CNTs, respectively (see Fig. 1). The 1 st CNT occupies the region $z \in\left[0, L_{1}\right]$ and the intertube contact occurs at the point with coordinate $z=r_{1}, 0<r_{1}<L_{1}$. The 2 nd CNT occupies the region $z_{2} \in\left[0, L_{2}\right]$ and the intertube contact takes place at $z_{2}=L_{2}-r_{2}, 0<r_{2}<L_{2}$.

The system of the crossing CNTs is exposed to an incident electromagnetic field with the axial components on the surface of the 1st and 2nd tubes being $E_{1 z}^{\mathrm{ex}}(z, t)=$ $E_{1 z}^{(0)}(z) \exp (-i \omega t)$ and $E_{2 z_{2}}^{\mathrm{ex}}\left(z_{2}, t\right)=E_{2 z_{2}}^{(0)}\left(z_{2}\right) \exp (-i \omega t)$, respectively. Here, $\omega$ is an angular frequency. Note also that $a_{m} \ll \lambda$, where $\lambda$ is a wavelength of the incident field. Furthermore, we shall consider the low frequency range $(<10 \mathrm{THz})$ where only intraband electron transitions are allowed in the CNTs. As these transitions contribute to the axial conductivity ${ }^{30}$, only the axial electric surface current density $j_{m}$ with polar symmetry are excited in the $m$-th CNT; then $j_{1}(\phi, z)=j_{1}(z)$, and $j_{2}\left(\phi_{2}, z_{2}\right)=j_{2}\left(z_{2}\right)$.

We shall model CNTs as finite length hollow cylinders with axial surface conductivity found from quantum mechanical considerations in the tight-binding approximation $^{1}$. For a small radius $(<2 \mathrm{~nm})$ metallic CNT, the surface conductivity is given by ${ }^{1}$

$$
\sigma_{m}(\omega)=\frac{2 i e^{2} v_{F}}{\pi^{2} \hbar a_{m}(\omega+i \nu)},
$$

where $v_{F}$ is the Fermi velocity for a CNT, $v_{F} \simeq 10^{6}$ $\mathrm{m} / \mathrm{s} ; \hbar$ is the reduced Planck constant; $e$ is the electron charge; $\nu$ is the electron relaxation frequency; $\nu=\tau^{-1}$, where $\tau$ is the electron relaxation time. We shall neglect the influence of the adjacent tube on the intrinsic tube conductivity $\sigma_{m}$ within the contact area, as this influence is rather weak ${ }^{29}$.

We suppose that the axial current on each tube has a polar symmetry even at the presence of intertube tunneling. Then the current on the 1st CNT satisfies the following boundary conditions at the contact point, $z=r_{1}$

$$
\begin{gathered}
j_{1}\left(r_{1}+0\right)-j_{1}\left(r_{1}-0\right)=I_{t} /\left(2 \pi a_{1}\right), \\
\left.\frac{\partial j_{1}}{\partial z}\right|_{z=r_{1}-0}=\left.\frac{\partial j_{1}}{\partial z}\right|_{z=r_{1}+0}
\end{gathered}
$$

where $I_{t}$ is an intertube tunneling current taken to be directed from the 2nd to the 1st CNT (see Fig. 2(a)). Condition (2) stems from the Kirchhoff's law while condition (3) expresses a continuity of the charge distribution at point $z=r_{1}$ (the relation between the charge and current densities is shown in Eq. (45)). The absence of concentrated charges on the 1st CNT edges must be ensured by the edge conditions for the current

$$
j_{1}(0)=j_{1}\left(L_{1}\right)=0
$$

Similar boundary conditions are true for the current $j_{2}$ 
on the 2nd CNT in the coordinate system $\left(\rho_{2}, \phi_{2}, z_{2}\right)$

$$
\begin{gathered}
j_{2}\left(L_{2}-r_{2}+0\right)-j_{2}\left(L_{2}-r_{2}-0\right)=-I_{t} /\left(2 \pi a_{2}\right), \\
\left.\frac{\partial j_{2}}{\partial z_{2}}\right|_{z_{2}=L_{2}-r_{2}-0}=\left.\frac{\partial j_{2}}{\partial z_{2}}\right|_{z_{2}=L_{2}-r_{2}+0} \\
j_{2}(0)=j_{2}\left(L_{2}\right)=0
\end{gathered}
$$

Let us note that the current discontinuity in (2) and (5) leads to the localization of charges $-q$ and $+q$, $q=i \omega^{-1} I_{t}$, at the point of junction on the 1 st and 2 nd CNTs, respectively. They are unphysical as their density is infinite. Those charges should be compensated in our model by the charges induced by intertube current $I_{t}$. Indeed, the intertube current $I_{t}$ can be modeled as a dipole with the charges $+q_{t}$ and $-q_{t}$, where

$$
q_{t}=i \omega^{-1} I_{t}
$$

The charges $+q_{t}$ and $-q_{t}$ are distributed on the infinitely thin rings of radius $a_{1}$ and $a_{2}$ on the surface of the 1st and 2nd CNTs, respectively, at the point of junction (see Fig. $2(\mathrm{a}, \mathrm{b}))$. In this case, the infinite charge density due to the current "jump" in CNTs is compensated completely by the charge density caused by the intertube current.

(a)

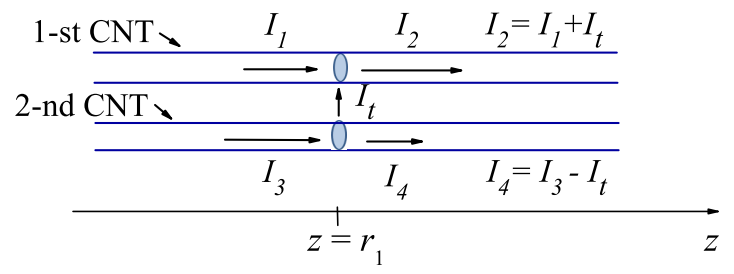

(b)

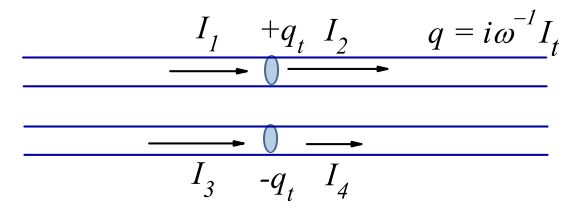

(c)

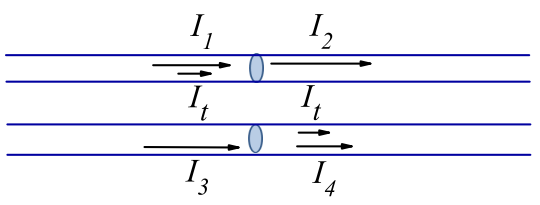

FIG. 2. (a) Scheme of two CNTs with the tunnel junction at $z=r_{1} . I_{t}$ is a current through the junction. $I_{1}, I_{2}, I_{3}$, and $I_{4}$ are currents in CNTs on the opposite sides in respect to the junction. For simplicity, tubes are shown to be parallel; in general, they could be non-parallel. (b) Same as (a) except, that the tunneling current element is replaced by the dipole with the charges $+q_{t}$ and $-q_{t}, q_{t}=i \omega^{-1} I_{t}$, on the CNT surface at $z=r_{1}$. (c) Same as (b) except, that the charges $+q_{t}$ and $-q_{t}$ are replaced by the azimuthally symmetrical homogenous axial current $I_{t}$ having a jump at $z=r_{1}$ from $I_{t}$ down to 0 in the 1 st CNT and from 0 up to $I_{t}$ in the 2 nd CNT, respectively.
We shall formulate the boundary value problem concerning only the 1st CNT in the coordinate system $(\rho, \phi, z)$; for the 2nd CNT, it can be done in the same way in the system $\left(\rho_{2}, \phi_{2}, z_{2}\right)$. The scattered electromagnetic field induced by the current $j_{1}$ can be expressed in terms of the electric Hertz potential $\Pi(\mathbf{r})=\Pi(\rho, z) \mathbf{e}_{z}$ which satisfies the Helmholtz equation:

$$
\left(\nabla^{2}+k^{2}\right) \Pi=0,
$$

where $\mathbf{e}_{z}$ is a unit vector along the $z$-axis, $k=\sqrt{\varepsilon_{h}} \omega / c$ is the wave number, and $c$ is the speed of light in vacuum. The scattered fields have the following non-zero components:

$E_{\rho}^{(1)}=\frac{\partial^{2} \Pi}{\partial z \partial \rho}, \quad E_{z}^{(1)}=\left(\frac{\partial^{2}}{\partial z^{2}}+k^{2}\right) \Pi, \quad H_{\phi}^{(1)}=i \varepsilon \omega \frac{\partial \Pi}{\partial \rho}$.

Expressions (10) can be used to find the field scattered by the 1 st CNT on the surface of the 2nd CNT.

The boundary conditions for Eq. (9) for the 1st CNT can be written as ${ }^{1}$

$$
\begin{gathered}
\left.\frac{\partial \Pi}{\partial \rho}\right|_{\rho=a_{1}+0}-\left.\frac{\partial \Pi}{\partial \rho}\right|_{\rho=a_{1}-0}=\frac{j_{1}(z)}{i \omega \varepsilon}, \quad z \in\left(0, L_{1}\right), \\
\left.\frac{\partial \Pi}{\partial \rho}\right|_{\rho=a_{1}+0}=\left.\frac{\partial \Pi}{\partial \rho}\right|_{\rho=a_{1}-0}, z \in(-\infty, 0) \cup\left(L_{1},+\infty\right), \\
\left.\Pi\right|_{\rho=a_{1}+0}=\left.\Pi\right|_{\rho=a_{1}-0}, \quad-\infty<z<+\infty .
\end{gathered}
$$

Let the electromagnetic field produced by the current $j_{2}$ be defined on the surface of the 1st tube as $\mathbf{E}_{1}^{(2)}\left(a_{1}, \phi, z\right)$. Since only azimuthally symmetric component of this field contributes to the axial current in the 1st CNT, we assume that $E_{1 z}^{(2)}\left(\rho=a_{1}, \phi, z\right)=E_{1 z}^{(2)}(\rho=$ $0, z) \equiv E_{1 z}^{(2)}(z)$. Similarly, let $E_{1 z}^{(1 t)}$ and $E_{1 z}^{(2 t)}$ be $z$ components of the electric fields induced on the 1st CNT by the charges $+q_{t}$ and $-q_{t}$, respectively. The total axial field on the surface of the 1st CNT is

$$
\begin{gathered}
E_{z}^{\mathrm{tot}}\left(a_{1}, z\right)=E_{1 z}^{(0)}(z)+E_{1 z}^{(2)}(z)+E_{1 z}^{(2 t)}(z)+E_{1 z}^{(1 t)}(z)+ \\
\left(\frac{\partial^{2}}{\partial z^{2}}+k^{2}\right) \Pi\left(a_{1}, z\right)
\end{gathered}
$$

where the last term is the field scattered by the 1st CNT (see $E_{z}^{(1)}$ in Eqs. (10)).

Surface current density on the surface of the 1st CNT in the local approximation satisfies the Ohm's law:

$$
j_{1}(z)=\sigma_{1} E_{z}^{\mathrm{tot}}\left(a_{1}, z\right)
$$

Intertube current can be found as

$$
I_{t}=G_{d}\left(U_{1}+U_{2}\right)
$$

where $G_{d}$ is the two-terminal intertube conductance that can be found from the four-terminal Landauer formalism (see Appendix A); $U_{1}\left(U_{2}\right)$ is intertube voltage caused by the current density $j_{1}\left(j_{2}\right)$ and charge $+q_{t}\left(-q_{t}\right)$. 
It has been found from measurements ${ }^{31}$ that the conductance of the intertube junction between metallic CNTs is in the range of $0.04 G_{0}-0.13 G_{0}$, where $G_{0}=$ $e^{2} /(\pi \hbar)$. Theoretical calculations predict that $G_{d}$ depends on the: (i) crossing angle ${ }^{32,33}$, (ii) contact distance and contact force ${ }^{29,34}$, (iii) interlinking bonds ${ }^{35}$, and (iv) presence of physisorbed molecules ${ }^{36}$ or linker atoms ${ }^{37}$.

To obtain the unique solution of the boundary value problem for two crossing CNTs, the boundary conditions must be supplemented by radiation conditions ${ }^{38}$.

\section{B. Pocklington type equation}

Let us now present a system of Pocklington type equations with the exact kernels for two crossing CNTs. The solution of Helmholtz equation (9) with boundary conditions (11) - (13) is sought as a single-layer potential for the field scattered by the current $j_{1}$ :

$$
\Pi(\rho, z)=\frac{i a_{1}}{\varepsilon \omega} \int_{0}^{L_{1}} j_{1}\left(z^{\prime}\right) G\left(\rho, z-z^{\prime}\right) d z^{\prime},
$$

where

$$
G(\rho, z)=\int_{0}^{2 \pi} \frac{\exp \left(i k \sqrt{a_{1}^{2}+\rho^{2}-2 \rho a_{1} \cos \phi+z^{2}}\right)}{4 \pi \sqrt{a_{1}^{2}+\rho^{2}-2 \rho a_{1} \cos \phi+z^{2}}} d \phi .
$$

Substitution of (17) into (14) allows one to obtain Pocklington integro-differential equation for the 1st CNT.

Let us first find a scalar potential $\varphi=-\partial \Pi / \partial z$ from (17). Replacing $\partial G / \partial z \rightarrow-\partial G / \partial z^{\prime}$, integrating by parts, and taking into account (2) and (3), we arrive at

$$
\begin{gathered}
\varphi(\rho, z)=-\frac{i a_{1}}{\varepsilon \omega}\left[j_{1}(0) G(\rho, z)-j_{1}\left(L_{1}\right) G\left(\rho, z-L_{1}\right)\right]- \\
\frac{i a_{1}}{\varepsilon \omega}\left(\int_{0}^{r_{1}-0}+\int_{r_{1}+0}^{L_{1}} \frac{\partial j_{1}\left(z^{\prime}\right)}{\partial z^{\prime}} G\left(\rho, z-z^{\prime}\right) d z^{\prime}\right)- \\
\frac{i a_{1}}{\varepsilon \omega}\left(j_{1}\left(r_{1}+0\right)-j_{1}\left(r_{1}-0\right)\right) G\left(\rho, z-r_{1}\right) .
\end{gathered}
$$

Because of the edge conditions (4), the terms with $j_{1}(0)$ and $j_{1}\left(L_{1}\right)$ equal zero in (19). The last term in (19), comprising $j_{1}\left(r_{1}+0\right)-j_{1}\left(r_{1}-0\right)$, determines the field produced by the charge $-q, q=i \omega^{-1} I_{t}$, which appears due the current discontinuity at $z=r_{1}$ according to (2). This term is equal in magnitude but opposite in sign to the potential $\varphi_{t}$ produced by the the charge $+q_{t}$ on the 1st CNT:

$$
\varphi_{t}(\rho, z)=\frac{i I_{t}}{2 \pi \varepsilon \omega} G(\rho, z-r 1)
$$

The axial electric field $E_{z}^{(1 t)}$ produced by the charge $+q_{t}$ is $E_{z}^{(1 t)}(\rho, z)=-\partial \varphi_{t}(\rho, z) / \partial z$. The intertube voltages $U_{1}$ can be found as follows

$U_{1}=\varphi\left(a_{1}+D, r_{1}\right)+\varphi_{t}\left(a_{1}+D, r_{1}\right)-\varphi\left(a_{1}, r_{1}\right)-\varphi_{t}\left(a_{1}, r_{1}\right)$.
Intertube voltage $U_{2}$ produced by the current $j_{2}$ and charge $-q_{t}$ can be found in a way analogous to (19)-(21). The axial field produced simultaneously by the current $j_{1}$ and charge $q_{t}$ is

$$
\begin{gathered}
\frac{\partial^{2} \Pi}{\partial z^{2}}+k^{2} \Pi+E_{z}^{(1 t)}=\frac{i a_{1}}{\varepsilon \omega}\left(\int_{0}^{r_{1}-0}+\int_{r_{1}+0}^{L_{1}} d z^{\prime} \times\right. \\
\left.\left[\frac{\partial j_{1}\left(z^{\prime}\right)}{\partial z^{\prime}} \frac{\partial G\left(\rho, z-z^{\prime}\right)}{\partial z}+k^{2} j_{1}\left(z^{\prime}\right) G\left(\rho, z-z^{\prime}\right)\right]\right) .
\end{gathered}
$$

After substitution of (22) into (14) at $\rho=a_{1}$ and taking into account (15), we arrive at the Pocklington type equation for the 1st CNT

$$
\begin{gathered}
\frac{j_{1}(z)}{\sigma_{1}}=E_{1 z}^{(0)}(z)+E_{1 z}^{(2)}(z)+E_{1 z}^{(2 t)}(z)+ \\
\frac{i a_{1}}{\varepsilon \omega}\left(\int_{0}^{r_{1}-0}+\int_{r_{1}+0}^{L_{1}} \frac{\partial j_{1}\left(z^{\prime}\right)}{\partial z^{\prime}} \frac{\partial G\left(a_{1}, z-z^{\prime}\right)}{\partial z} d z^{\prime}\right)+ \\
\frac{i a_{1}}{\varepsilon \omega} \int_{0}^{L_{1}} k^{2} j_{1}\left(z^{\prime}\right) G\left(a_{1}, z-z^{\prime}\right) d z^{\prime} .
\end{gathered}
$$

The field $E_{1 z}^{(2)}+E_{z}^{(2 t)}$ produced by the current $j_{2}$ and charge $-q_{t}$ can be found by analogy to (22). Equation (23) must be supplemented by Eq. (16), Pocklington type equation (B1) for the 2nd CNT (see Appendix B), and boundary conditions (2)-(7). In solving the system of the resulting equations (23) and (B1), we used suitably smooth sub-domain basis functions for the current expansion, though the application of pulse functions is also possible ${ }^{39}$.

\section{Hallén type equation}

Toward an efficient numerical solution at lower frequencies, we formulated the scattering problem using the Hallén type equations. Let us note that the forth and fifth terms in (14) tend to infinity at $z \rightarrow r_{1}$, that leads to difficulties in a numerical solution of Hallén type equation describing the boundary value problem. Let us also notice that the sum of the the forth and fifth terms in (14) is finite. To avoid infinite terms in (14), we replace in our task the charges $+q_{t}$ and $-q_{t}$ by the following surface current densities $j_{1 a}\left(\rho=a_{1}, z\right) \equiv j_{1 a}(z)$ and $j_{2 a}\left(\rho_{2}=a_{2}, z_{2}\right) \equiv j_{2 a}\left(z_{2}\right)$ on the surface of infinite-length cylinders which coincide with the 1st and 2nd CNTs, respectively (see Fig. 2(b,c)),

$$
\begin{gathered}
j_{1 a}(z)=\left\{\begin{array}{l}
I_{t} /\left(2 \pi a_{1}\right), z \in\left(-\infty, r_{1}\right) \\
0, z \in\left(r_{1},+\infty\right)
\end{array},\right. \\
j_{2 a}\left(z_{2}\right)=\left\{\begin{array}{l}
0, z_{2} \in\left(-\infty, L_{2}-r_{2}\right) \\
I_{t} /\left(2 \pi a_{2}\right), z_{2} \in\left(L_{2}-r_{2},+\infty\right)
\end{array}\right.
\end{gathered}
$$

This replacement is justified for electrically small system of CNTs $\left(L_{1}+L_{2} \ll \lambda\right)$ as the current densities $j_{1 a}$ and $j_{2 a}$ produce the same scalar field potentials as 
charges $+q_{t}$ and $-q_{t}$, respectively (see Appendix C). As discussed in Appendix $\mathrm{C}$, there is some arbitrariness in choosing the currents $j_{1 a, 2 a}$. Then, the total axial field on the surface of the 1st CNT $\left(z \in\left[0, L_{1}\right]\right)$ can be expressed as

$$
E_{z}^{\mathrm{tot}}\left(a_{1}, z\right) \cong E_{1 z}^{(0)}(z)+\tilde{E}_{1 z}^{(2)}(z)+\left(\frac{\partial^{2}}{\partial z^{2}}+k^{2}\right) \tilde{\Pi}\left(a_{1}, z\right)
$$

where $\tilde{E}_{1 z}^{(2)}(z)$ is an electric field produced by $j_{2}+j_{2 a}$, $\tilde{\Pi}$ is a Hertz potential of the field produced by the current density $j_{1}+j_{1 a} ; \tilde{\Pi}$ satisfies Helmholtz equation and boundary conditions

$$
\begin{gathered}
\left.\frac{\partial \tilde{\Pi}}{\partial \rho}\right|_{\rho=a_{1}+0}-\left.\frac{\partial \tilde{\Pi}}{\partial \rho}\right|_{\rho=a_{1}-0}= \\
\frac{1}{i \omega \varepsilon}\left\{\begin{array}{l}
j_{1}+j_{1 a}, z \in\left[0, L_{1}\right] \\
j_{1 a}, z \in(-\infty, 0) \cup\left(L_{1},+\infty\right)
\end{array}\right. \\
\left.\tilde{\Pi}\right|_{\rho=a_{1}+0}=\left.\tilde{\Pi}\right|_{\rho=a_{1}-0}, \quad-\infty<z<+\infty .
\end{gathered}
$$

Taking into account the relation between the electric potential $\varphi$ and Hertz potential $\tilde{\Pi}, \varphi=-\partial \tilde{\Pi} / \partial z$, one can obtain the intertube voltage

$$
\left.U_{1} \approx \frac{\partial}{\partial z}\left[\tilde{\Pi}\left(a_{1}, z\right)-\tilde{\Pi}\left(a_{1}+D, z\right)\right]\right|_{z=r_{1}} .
$$

The second component of the intertube voltage $U_{2}$ can be found in the same way from the field induced by $j_{2}$ and $j_{2 a}$.

Thus, the impact of the tunneling current on the current in the tubes could be taken into account by means of the boundary conditions (2) and (5) and introduction of the extra currents (24) and (25).

The solution of Helmholtz equation for $\tilde{\Pi}$ with boundary conditions (27) - (28), taking into account (24), is sought as a single-layer potential

$$
\begin{gathered}
\tilde{\Pi}(\rho, z)=\frac{i a_{1}}{\varepsilon \omega}\left(\int_{0}^{L_{1}} j_{1}\left(z^{\prime}\right) G\left(\rho, z-z^{\prime}\right) d z^{\prime}+\right. \\
\left.\frac{I_{t}}{2 \pi a_{1}} \int_{-\infty}^{r_{1}} G\left(\rho, z-z^{\prime}\right) d z^{\prime}\right),
\end{gathered}
$$

where $j_{1}(z)$ and $I_{t}$ are the unknown surface current density and the intertube tunnel current to be found.

The potential $\tilde{\Pi}\left(a_{1}, z\right)$ can be expressed from (26) as follows

$$
\tilde{\Pi}\left(a_{1}, z\right)=\int_{0}^{L_{1}} E_{z}^{\mathrm{tot}}\left(a_{1}, z\right) \mathrm{e}^{i k\left|z-z^{\prime}\right|} d z^{\prime}-\Phi(z),
$$

where

$$
\begin{aligned}
\Phi(z)= & \int_{0}^{L_{1}}\left(E_{1 z}^{(0)}(z)+\tilde{E}_{1 z}^{(2)}(z)\right) \mathrm{e}^{i k\left|z-z^{\prime}\right|} d z^{\prime}+ \\
& \left\{\begin{array}{l}
C_{1} \mathrm{e}^{i k z}+C_{2} \mathrm{e}^{-i k z}, z \in\left(0, r_{1}\right) \\
D_{1} \mathrm{e}^{i k z}+D_{2} \mathrm{e}^{-i k z}, z \in\left(r_{1}, L_{1}\right)
\end{array}\right.
\end{aligned}
$$

with $C_{1,2}$ and $D_{1,2}$ being unknown constants to be determined from the edge conditions (2)-(4).

After substitution of (31) into (30) at $\rho=a_{1}$, and taking into account (15), we arrive at the Hallén equation for the 1st CNT

$$
\begin{gathered}
\int_{0}^{L_{1}}\left[\sigma_{1}^{-1} \mathrm{e}^{i k\left|z-z^{\prime}\right|}+\frac{2 a_{1} k}{\varepsilon \omega} G\left(a_{1}, z-z^{\prime}\right)\right] j_{1}\left(z^{\prime}\right) d z^{\prime}+ \\
\frac{k I_{t}}{\pi \varepsilon \omega} \int_{-\infty}^{r_{1}} G\left(a_{1}, z-z^{\prime}\right) d z^{\prime}=\Phi(z)
\end{gathered}
$$

Let us notice that the form of the Hallén equation (33) depends on the choice of the current $j_{1 a}$. Equation (33) must be supplemented by Eq. (16) and Hallén equation (B2) for the 2nd CNT (see Appendix B).

The integrals in (33) and (B2) can be handled by a quadrature formula, thereby transforming (33) and (B2) into matrix equations. As the current varies significantly near the crossing point, the discretization step along the $z$ axis should be comparable with tube diameter in the vicinity of this point. The matrix equations can be solved numerically to find the current density in the CNTs and intertube current $I_{t}$. In solving numerically the Halléntype integral equations by the collocation method we arrive at a matrix that is close to degenerate at low frequencies. This leads to instability of the numerical matrix inversion in the quasistatic regime.

We compared the results given by Hallén and Pocklington equations and found a good agreement within the error of numerical computations $(<1 \%)$ for data presented in Fig. 6. We also found that the Pocklington equation gives a stable solution in a wide frequency range $(>1$ $\mathrm{MHz})$, whereas Hallén equation produces stable results only at high frequencies $(>50 \mathrm{GHz})$ for the parameters of the crossing tubes same as in Fig. 7. Results presented below in Sec. III have been obtained at high frequencies using Hallén equation (Figs. 3-5,9,10) and at low frequencies using Pocklington equation (Figs. 6-8).

\section{Superposition of CNT currents and introduction of the equivalent RC-circuit}

Consider two crossed CNTs exposed to the incident arbitrary-oriented electromagnetic field with electric field magnitude $E^{(0)}$. Let the current densities $j_{1,2}$ and intertube current $I_{t}$ be known from the solution of Eqs. (33), (B2) and (16). As follows from (33) and (B2), the current densities $j_{1,2}$ can be divided into two parts

$$
j_{1}(z)=j_{1}^{(e)}(z)+j_{1}^{(c)}(z), \quad j_{2}\left(z_{2}\right)=j_{2}^{(e)}\left(z_{2}\right)+j_{2}^{(c)}\left(z_{2}\right)
$$

where the terms $j_{1,2}^{(e)}$ satisfy (33), (B2) and (2)-(4) with $I_{t}=0$; the terms $j_{1,2}^{(c)}$ satisfy the same equations but with $E_{1 z}^{(0)}(z)=E_{2 z_{2}}^{(0)}\left(z_{2}\right)=0$ and $I_{t} \neq 0$. The current densities $j_{1,2}^{(e)}(z)$ describe the CNT responses to the external field when $G_{d}=0$. The current densities $j_{1,2}^{(c)}(z)$ describe the 
transport of the charges passing between the CNTs due to the tunneling current $I_{t}$.

Since $j_{1,2}^{(e)} \propto E^{(0)}$ and $j_{1,2}^{(c)} \propto I_{t}$, the current densities $j_{1,2}$ in (34) can be represented through normalized current densities $j_{1 n, 2 n}^{(e, c)}$ as follows

$$
\begin{aligned}
& j_{1}(z)=j_{1 n}^{(e)}(z) E^{(0)}+j_{1 n}^{(c)}(z) I_{t}, \\
& j_{2}\left(z_{2}\right)=j_{2 n}^{(e)}\left(z_{2}\right) E^{(0)}+j_{2 n}^{(c)}\left(z_{2}\right) I_{t} .
\end{aligned}
$$

Then the intertube voltage $U_{1}+U_{2}$ produced by the currents $j_{1}$ and $j_{2}$ can be divided into two components

$$
U_{1}+U_{2}=P E^{(0)}-K I_{t},
$$

where $P$ and $K$ are the proportionality coefficients. The term $P E^{(0)}$ in $(37)$ is a voltage produced by the currents $j_{1,2}^{(e)}$, it can be considered as the external voltage applied to the intertube contact. The term $-K I_{t}$ in (37) is a voltage produced by the currents $j_{1,2}^{(c)}$; it can be interpreted as the depolarizing voltage induced by the charges transferred between the tubes.

Our numerical calculations show that the frequency dependencies of the coefficients $K$ and $P$ can be approximated with high accuracy in the range below the frequencies of the LPR and electron relaxation frequency $\nu$ in the 1st and 2nd CNTs as follows:

$$
\begin{gathered}
K(\omega) \approx K^{\prime}+i \omega^{-1} K^{\prime \prime}, \\
P(\omega) \approx P^{\prime}+i \omega P^{\prime \prime},
\end{gathered}
$$

where $K^{\prime}, K^{\prime \prime}, P^{\prime}$, and $P^{\prime \prime}$ are real positive values. Note that $\operatorname{Im}(P) \ll \operatorname{Re}(P)$ and $\operatorname{Im}(K) \gg \operatorname{Re}(K)$. For realistic values of $G_{d}$, the inequality $G_{d}^{-1} \gg \operatorname{Re}(K)$ is true.

After substitution of (38) and (39) into (37) and substitution of (37) into (16), one can express the current $I_{t}$ from (16) as follows:

$$
I_{t} \approx \frac{\left(P^{\prime}+i \omega P^{\prime \prime}\right) E^{(0)}}{G_{d}^{-1}+K^{\prime}+i \omega^{-1} K^{\prime \prime}} .
$$

Equation (40) is true in the frequency range below the LPR peak. It can be considered as an expression for the current in the equivalent RC-circuit (or RC-filter) composed of a resistor with the resistance $R=G_{d}^{-1}+K^{\prime}$ and a capacitor with the capacitance $C=1 / K^{\prime \prime}$; frequency dependent voltage $U_{0}=\left(P^{\prime}+i \omega P^{\prime \prime}\right) E^{(0)}$ is applied to this RC-circuit. The circuit has a cutoff frequency $f_{c}=(2 \pi R C)^{-1}$ defining two response regimes: (i) at $f \ll f_{c}$, the capacitor has sufficient time to charge up, so that the current $I_{t}$ is strongly suppressed by the capacity voltage; hereafter, this regime will be called a regime of the high-transparency of the intertube junction; (ii) at $f \gg f_{c}$, the capacitor has insufficient time to charge up, so that the current $I_{t}$ is determined mainly by the external voltage $U_{0}$; this regime will be referred as a regime of the low-transparency of the intertube junction. Both regimes will be considered in Sec. III B.

\section{E. Electromagnetic parameters of CNTs}

Let $\mathbf{d}_{m}=d_{m} \mathbf{e}_{m}$ be a dipole moment of the $m$-th CNT, where $\mathbf{e}_{m}$ is a unit vector along the $m$-th CNT, $m=1,2$, and let $\mathbf{E}^{(0)}$ be a vector of the incident field. Let us define a polarizability tensor $\hat{\alpha}$ of the system of the crossing CNTs as follows

$$
\mathbf{d}_{1}+\mathbf{d}_{2}=\hat{\alpha} \mathbf{E}^{(0)}
$$

Then

$$
\hat{\alpha}=\alpha_{11} \mathbf{e}_{1} \otimes \mathbf{e}_{1}+\alpha_{12} \mathbf{e}_{1} \otimes \mathbf{e}_{2}+\alpha_{21} \mathbf{e}_{2} \otimes \mathbf{e}_{1}+\alpha_{22} \mathbf{e}_{2} \otimes \mathbf{e}_{2},
$$

where $\alpha_{m n}(m, n=1,2)$ are parameters that relate the magnitude of dipole moments and projections of the incident field on the CNT axes

$$
\left[\begin{array}{l}
d_{1} \\
d_{2}
\end{array}\right]=\left[\begin{array}{ll}
\alpha_{11} & \alpha_{12} \\
\alpha_{21} & \alpha_{22}
\end{array}\right]\left[\begin{array}{l}
\mathbf{E}^{(0)} \cdot \mathbf{e}_{1} \\
\mathbf{E}^{(0)} \cdot \mathbf{e}_{2}
\end{array}\right] .
$$

Once we know the surface current density, we can calculate the magnitude of the dipole moments of $m$-th CNT in the long-wavelength regime $\left(L_{1}+L_{2} \ll \lambda\right)$

$$
d_{m}=\frac{2 \pi i a_{m}}{\omega} \int_{0}^{L_{m}} j_{m}(z) d z, \quad m=1,2 .
$$

For further analysis, we shall consider a symmetric geometry providing excitation of similar currents in both tubes, i.e. $L_{1}=L_{2}=L, \sigma_{1}=\sigma_{2}, r_{1}=r_{2}=r<L / 2$, $E_{1 z}^{(0)}= \pm E_{2 z_{2}}^{(0)}$. Then $\alpha_{11}=\alpha_{22}, \alpha_{12}=\alpha_{21}$, and $d_{1}= \pm d_{2}$. We shall refer to the value $\alpha_{1 z}=d_{1} / E_{1 z}^{(0)}=$ $\alpha_{11} \pm \alpha_{12}$ as the polarizability of the 1 st CNT that takes place at $E_{1 z}^{(0)}= \pm E_{2 z_{2}}^{(0)}$.

The surface charge density on the 1st CNT can be found from the continuity equation as

$$
\rho_{c 1}(z)=-\frac{i}{\omega} \frac{\partial j_{1}(z)}{\partial z}, \quad z \in\left(0, L_{1}\right)
$$

The power $P_{c n}$ dissipated in the crossed CNTs by the currents $j_{1}$ and $j_{2}$ can be found as follows ${ }^{12}$ :

$$
P_{c n}=\pi \sum_{m=1,2} a_{m} \operatorname{Re}\left(\sigma_{m}^{-1}\right) \int_{0}^{L_{m}}\left|j_{m}(z)\right|^{2} d z .
$$

The dissipated power $P_{c}$ caused by the tunneling current $I_{t}$ can be obtained as ${ }^{40}$ :

$$
P_{c}=\frac{\left|I_{t}\right|^{2}}{2 G_{d}}
$$

The power $P_{c}$ is dissipated within a distance of the order of the electronic mean free path from the intertube junction. 


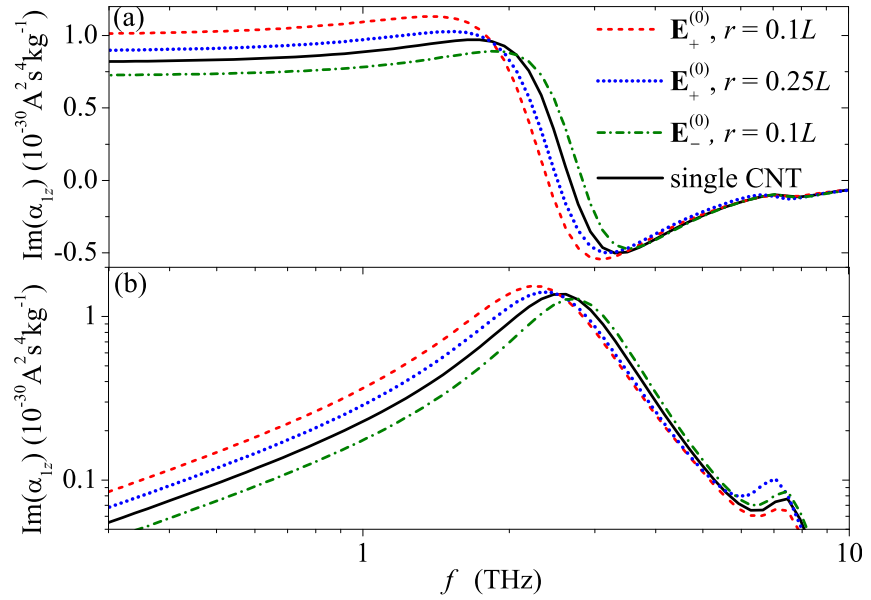

FIG. 3. Frequency dependence of (a) the real and (b) imaginary parts of the polarizability of the 1st CNT at different distances $r=0.1,0.25 \mathrm{~L}$ and different incident fields $\mathbf{E}_{+}^{(0)}$ and $\mathbf{E}_{-}^{(0)} ; \theta=45^{\circ}$. There is no current between the tubes, i.e. $G_{d}=0$. The polarizability of single CNT is shown by solid line.

\section{NUMERICAL RESULTS AND DISCUSSION}

In our calculations, we consider two identical zigzag $(15,0)$ CNTs of length $L=1 \mu \mathrm{m}$ crossing as shown in Fig. 1 at angle $\theta \leq 90^{\circ}$ and $r \leq L / 2$. For calculation of the tube conductivity, we shall use an electron relaxation time of $\tau=100 \mathrm{fs}^{27}$.

We shall consider two orientations of the incident field, as shown in Fig. 1: (i) $E_{1 z}^{(0)}=E_{2 z_{2}}^{(0)}=3 \times 10^{4} \mathrm{~V} / \mathrm{m}$ (electric field $\mathbf{E}_{+}^{(0)}$ ) and (ii) $E_{1 z}^{(0)}=-E_{2 z_{2}}^{(0)}=3 \times 10^{4} \mathrm{~V} / \mathrm{m}$ (electric field $\mathbf{E}_{-}^{(0)}$ ). Here, we neglect the variation of the incident field within the CNT system, as the longwavelength regime is valid $(\lambda \gg L)$. Because of the mirror symmetry of the problem geometry and the symmetry of the incident field, the current and charge densities in the tubes have the following symmetry $j_{1}(z)=j_{2}\left(L-z_{2}\right)$, $\rho_{c 1}(z)=-\rho_{c 2}\left(L-z_{2}\right)$ if $z=z_{2}$; here the current density $j_{1,2}$ and charge density $\rho_{c 1, c 2}$ on the 1st and 2nd CNTs are considered in coordinate systems with axises $z$ and $z_{2}$, respectively, as shown in Fig. 1. Then, the polarizabily is the same for these tubes, and we shall present the parameters $\alpha_{1 z}, j_{1}$ and $\rho_{c 1}$ only for the 1st CNT. For comparison, we shall also reproduce data for a single 1 $\mu \mathrm{m}$ long zigzag $(15,0) \mathrm{CNT}$.

\section{A. Crossing CNTs with zero intertube conductance}

Let us first assume that there is only electromagnetic interaction between the tubes; the intertube current equals zero $\left(G_{d}=0\right)$. Figure 3 shows the frequency dependences of the real and imaginary parts of the polarizability $\alpha_{1 z}$ in the range $0.1-10 \mathrm{THz}$ for the 1 st CNT at different positions of the crossing point $r$ and different orientations of the incident field, $\mathbf{E}_{+}^{(0)}$. For comparison, the polarizability of a single CNT is presented in Fig. 3 by solid line. All the spectra in Fig. 3(b) contain a broad peak at $\approx 2.5 \mathrm{THz}$ due to the first antenna (or localized plazmon) resonance in finite-length $\mathrm{CNT}^{4}$.
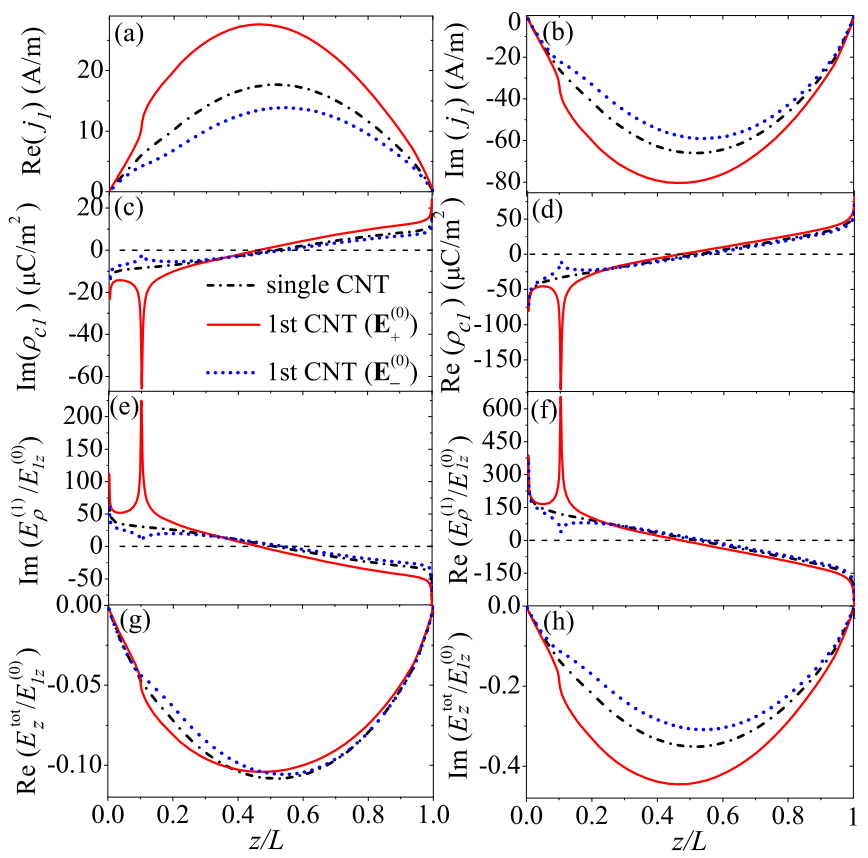

FIG. 4. Distribution of (a,b) the surface current density, (c,d) charge-density, $(\mathrm{e}, \mathrm{f})$ radial and $(\mathrm{g}, \mathrm{h})$ axial fields on the surface of the 1 st $\mathrm{CNT}$ and single CNT at $1 \mathrm{THz} ; G_{d}=0, \theta=45^{\circ}$, and $r=0.1 L$.

As shown in Fig. 3(b), the electromagnetic interaction between the tubes leads (i) to a red shift of the LPR peak from $2.53 \mathrm{THz}$ to $2.26 \mathrm{THz}$ for the incident field $\mathbf{E}_{+}^{(0)}$ and (ii) to the blue shift of this peak from $2.53 \mathrm{THz}$ to 2.69 $\mathrm{THz}$ for the incident field $\mathbf{E}_{-}^{(0)}$.

Physical mechanism of the electromagnetic interaction is clear from Fig. 4 where the current density, charge density, as well as radial and axial fields on the CNT surface are shown for the single tube and for the 1st CNT for different polarizations of the incident field, $\mathbf{E}_{ \pm}^{(0)}$, and $f=1$ $\mathrm{THz}$. Due to the polarization effect, there is high density charge distribution along the CNT (see Fig. 4(c,d)). For the external field $\mathbf{E}_{+}^{(0)}$, the unlike charges are concentrated on the 1 st and 2 nd tubes near the crossing point. Attraction between these charges leads to a sharp peak in the total charge distribution at $z / L=0.1$ (Fig. $4(\mathrm{c}, \mathrm{d})$ ). This results in the enhancement of the total field in the intertube gap (see a sharp peak at $z / L=0.1$ in the radial field distribution of the 1st CNT in Fig. 4(e,f)). The energy of the Coulomb interaction of unlike charges near the crossing point is negative resulting in the red shift of the LPR peak in comparison with the LPR peak of a 
single CNT (see Fig. 3(b)). This shift increases with the charge density near the crossing point. Since the charge density is maximal at minimal values of $r$, the LPR peak shift is larger for smaller values of $r$. This tendency is demonstrated in Fig. 3 (compare the spectra for $\mathbf{E}_{+}^{(0)}$ at $r=0.1 L$ and $r=0.25 L)$.

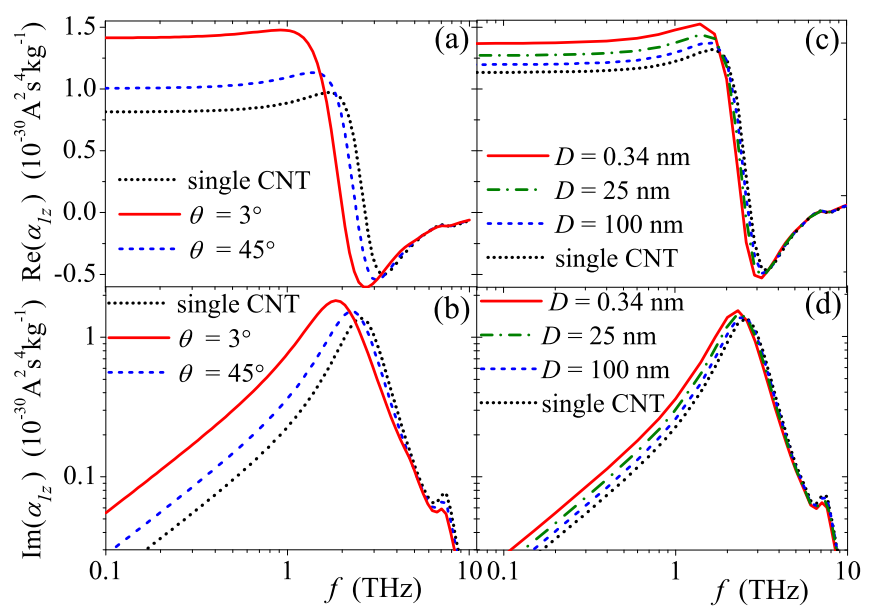

FIG. 5. Frequency dependence of the polarizability $\alpha_{1 z}$ of the 1st CNTs for various (a,b) angles between CNTs $\theta=3^{\circ}, 45^{\circ}$, $D=0.34 \mathrm{~nm}$ and (c,d) distances $D \in\{0.34,25,100\} \mathrm{nm}$, $\theta=45^{\circ} ; r=100 \mathrm{~nm} ; G_{d}=0$. The polarizability of a single CNT is shown by the dotted line. The external field is $\mathbf{E}_{+}^{(0)}$.

For the incident field $\mathbf{E}_{-}^{(0)}$, the like charges are accumulated on the 1st and 2nd tubes near the crossing point. Repulsion between these charges leads to a dip in the total charge distribution at $z / L=0.1$ (see Fig. $4(\mathrm{c}, \mathrm{d})$ ). The radial field produced by the charge on each tube becomes weak at the crossing point (see Fig. 4(e,f)). Since the energy of the Coulomb interaction between the like charges near the crossing point has positive sign, the blue shift of the LPR peak occurs in this case (see Fig. 3(b)). The blue shift is typically smaller than the red one, as the charge variation near the crossing point due to the intertube interaction is smaller for the incident field $\mathbf{E}_{-}^{(0)}$ than for $\mathbf{E}_{+}^{(0)}$. Thus, the electromagnetic interaction can shift the LPR peak to higher or lower frequencies depending on the direction of the incident field.

The existence of two plasmon modes has been reported for a metallic nanoparticle dimer ${ }^{41-44}$. It has been shown that such a dimer supports a bonding plasmon at lower frequencies and an anti-bonding plasmon at higher frequencies. Bonding (anti-bonding) plasmon occurs when both particles are polarized in the same (opposite) directions. For the crossing CNTs, the incident fields $\mathbf{E}_{+}^{(0)}$ and $\mathbf{E}_{-}^{(0)}$ lead to the excitation of the collective plasmon modes corresponding to the bonding and anti-bonding plasmon modes, respectively.

Figure 5 shows the imaginary part of the polarizability $\alpha_{1 z}$ of the 1 st CNT at different angles $\theta$ and distances $D$ between CNTs for the incident field $\mathbf{E}_{+}^{(0)}$. For comparison, the spectrum of a single CNT is presented by a dotted line. One can see from Fig. 5 that the red shift of the LPR peak is not strong in comparison with the peak width, and it becomes weaker if the angle $\theta$ and distance $D$ increase. The larger the distance $D$ and angle $\theta$ the weaker is the interaction between the charges at the crossing point and consequently, the smaller the shift of the LPR peak. For example, as shown in Fig. 5(b), the electromagnetic interaction between the tubes leads to the peak shift from $1.85 \mathrm{THz}$ to $2.21 \mathrm{THz}$ due to angle variation from $3^{\circ}$ to $45^{\circ}$. Figure $5(\mathrm{c}, \mathrm{d})$ demonstrates that the maximal red shift of the LPR peak occurs at minimal value of $D=0.34 \mathrm{~nm}$, and it vanishes when the distance $D$ exceeds $100 \mathrm{~nm}$.

Thus, the electromagnetic coupling of the crossing CNTs occurs by means of the Coulomb interaction of the charges located near the crossing point. This interaction is rather weak resulting in a slight variation of the tube polarizability. Since the intertube voltage is maximal for the incident field $\mathbf{E}_{+}^{(0)}$, in the next section, we shall consider only this case taking into account intertube electron transport.

\section{B. Crossing CNTs with non-zero intertube transport}

Figure 6 shows the frequency dependence of the polarizabilities of a single CNT and the 1st CNT for various intertube conductances. The spectrum of the imaginary part of the polarizability $\operatorname{Im}\left(\alpha_{1 z}\right)$ has two peaks: broad gigahertz peak located in the range $1-700 \mathrm{GHz}$ and LPR peak appearing in the terahertz range 1-4 THz. The center frequency of the gigahertz peak increases with increasing the intertube conductance $G_{d}$, whereas the LPR peak at $2.3 \mathrm{THz}$ practically does not depend on the value of $G_{d}$. Additionally, due to the intertube tunneling current, the imaginary part of the polarizability increases drastically (by a factor 10 or even 100) below $1 \mathrm{THz}$, whereas the real part of the polarizability increases by a factor of about 2.1. It should be noticed that two crossing tubes do not behave as a single longer tube, however their response is qualitatively similar to that of a CNT with a short low-conductive section ${ }^{8}$.

For deeper understanding of the mechanism of the electromagnetic interaction of two crossing CNTs, we represented in Fig. 7 the spectra of the power $P_{c n}$ dissipated by the currents $j_{1,2}$ and power $P_{c}$ dissipated by the intertube current $I_{d}$ for the case of $G_{d}=0.1 G_{0}$. As shown in Fig. 7, the inequality $P_{c}>P_{c n}$ is true below $0.4 \mathrm{THz}$ thus explaining the high value of $\operatorname{Im}\left(\alpha_{1 z}\right)$ in this range. Moreover, the charge transfer between the tubes leads to a reduction of the depolarizing field along the tubes resulting in an increase of the value $P_{c n}$ (compare $P_{c n}$ at $G_{d}=0.1 G_{0}$ and $G_{d}=0$ below $0.4 \mathrm{THz}$ in Fig. 7).

Below the LPR peak $(f<2 \mathrm{THz})$, the crossing CNTs can be considered as a capacitor. The intertube current 


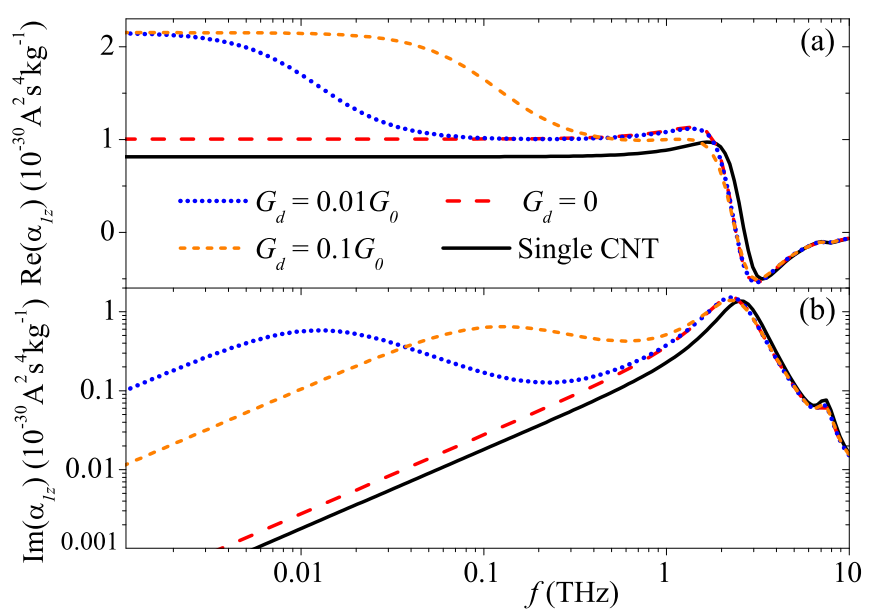

FIG. 6. Frequency dependence of (a) the real and (b) imaginary parts of the polarizability of the 1 st CNT for various intertube conductances $G_{d} \in\{0,0.01,0.1\} G_{0} ; \theta=45^{\circ}$; $r=L / 10$. The polarizability of a single CNT is shown by solid line.

can be described by Eq. (40) for RC-circuit (see Sec. IID). The cutoff frequency $f_{c}$ of the RC-circuit corresponds to the frequency of the gigahertz peak in Fig. $6(\mathrm{~b})$. Let us indicate three regimes of the intertube tunneling for the case of $G_{d}=0.1 G_{0}$ :

(i) Regime of the high-transparency of the intertube junction, $f<30 \mathrm{GHz}$. At low frequencies $\left(f \ll f_{c}\right.$, $f_{c}=0.1 \mathrm{THz}$ ), the incident field oscillates quite slowly, so that the charge carriers have enough time to pass through the intertube junction and be redistributed between the tubes. For this regime, the approximate equality $\operatorname{Re}\left[\alpha_{1 z}(f)\right] \approx \operatorname{Re}\left[\alpha_{1 z}(0)\right]$ is true. Figure 8 demonstrates the distributions of the current density $j_{1}$ and charge density $\rho_{c 1}$ along the 1 st CNT at $20 \mathrm{GHz}$. Current density components $j_{1}^{(e, c)}$ obtained in accordance with (34) and corresponding charge densities $\rho_{c 1}^{(e, c)}=$ $-i \omega^{-1} \partial j_{1}^{(e, c)} / \partial z$ are shown in Fig. 8. The charge with the density $\rho_{c 1}^{(e)}$ oscillates along the 1 st CNT and creates "external" field in the gap between the tubes, while the charge passing between the tubes spreads along the 1st CNT with the density $\rho_{c 1}^{(c)}$ and creates the "depolarizing" field in the gap. Distribution of the charge density $\rho_{c 1}^{(c)}$ is almost homogenous along the tube except near the tube edges and crossing point (see Fig. 8(c,d)). Due to the strong depolarization effect, resulting charge density $\rho_{c 1}=\rho_{c 1}^{(c)}+\rho_{c 1}^{(e)}$ at the crossing point is small (see Fig. $8(\mathrm{c}, \mathrm{d}))$, the intertube votage is low, and the intertube current is suppressed.

(ii) Intermediate regime, $f \in(30,500) \mathrm{GHz}$. The depolarizing field in the gap between the tubes decreases as frequency increases. Though the current $j_{1}^{(c)}$ becomes smaller, it is still comparable with $j_{1}^{(e)}$, and the intertube current $I_{d}$ reaches its maximum at $f \approx f_{c}$.

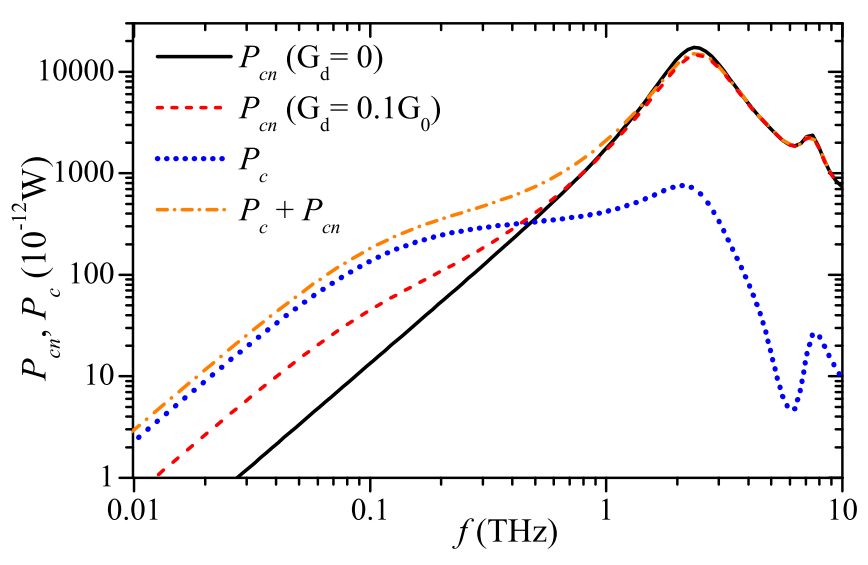

FIG. 7. Frequency dependencies of the dissipated powers $P_{c n}$, $P_{c}$, and $P_{c n}+P_{c}$ at $G_{d}=0.1 G_{0}$ and dissipated power $P_{c n}$ at $G_{d}=0 ; \theta=45^{\circ} ; r=L / 10$.

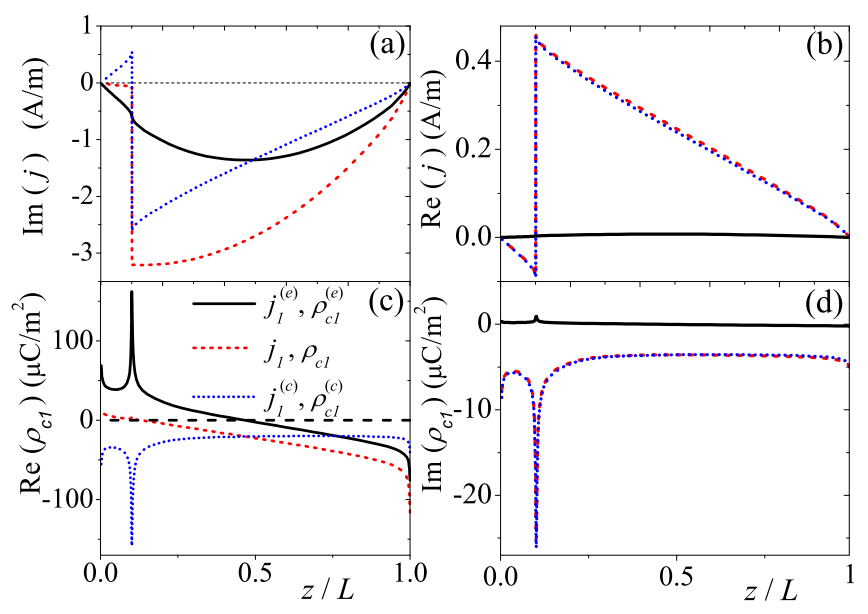

FIG. 8. Distribution of (a,b) the surface current density $j_{1}$ and $(\mathrm{c}, \mathrm{d})$ surface charge density $\rho_{c 1}$ and their components $j_{1}^{(c, e)}$ and $\rho_{c 1}^{(c, e)}$ on the surface of the 1 st CNT at $f=20 \mathrm{GHz}$; $G_{d}=0.1 G_{0} ; \theta=45^{\circ} ; r=L / 10$.

(iii) Regime of the low-transparency of the intertube junction, $f>500 \mathrm{GHz}$. At high frequencies $\left(f \gg f_{c}\right)$, the incident field oscillates quite quickly, so that small amount of charge can be transferred between the tubes during the period of the field oscillations. In this range, the value of $\operatorname{Re}\left[\alpha_{1 z}\right]$ is close to that for $G_{d}=0$ (see Fig. $6(\mathrm{a}))$. The total field in the gap between the tubes is determined mainly by the charge density $\rho_{c 1}^{(e)}$, so that the magnitude of intertube voltage and, consequently, the dissipated power in the gap $P_{c}$ practically do not depend on frequency in the range between the gigahertz and terahertz peaks (see Fig. 7). However the charge density $\rho_{c 1}^{(c)}$ and current density $j_{1}^{(c)}$ still should be taken into account, as they contributes significantly into the values of $\operatorname{Im}\left(\alpha_{1 \mathrm{z}}\right)$ and $P_{c}$ in the range below $1 \mathrm{THz}$.

Localized plasmon resonance in the CNTs manifests it- 


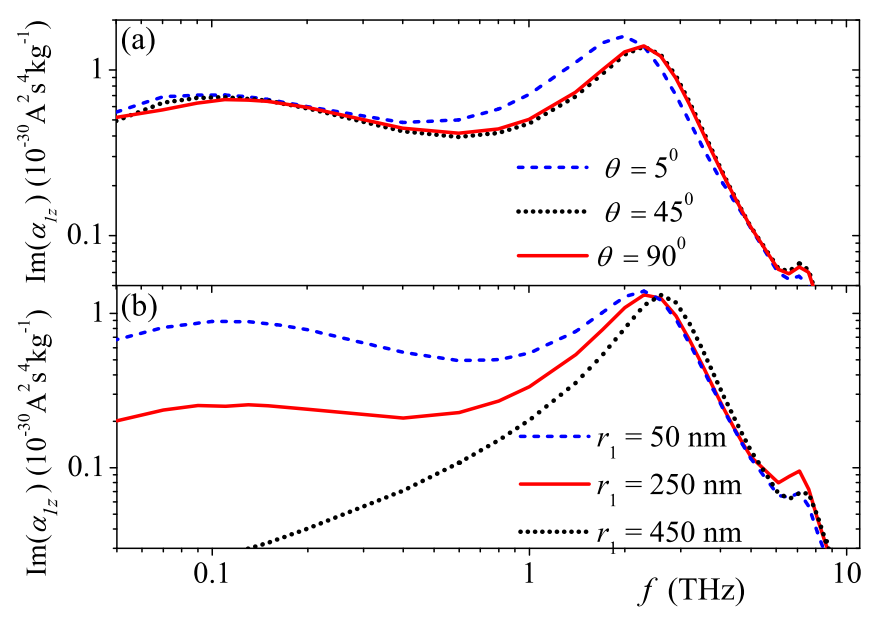

FIG. 9. Frequency dependence of the imaginary part of the polarizability of the 1st CNT for (a) various angles between the crossing tubes $\theta \in\left\{3^{\circ}, 45^{\circ}, 90^{\circ}\right\}, r=100 \mathrm{~nm}$, and (b) various distances $r \in\{50,250,450\} \mathrm{nm}, \theta=45^{\circ} ; G_{d}=0.1 G_{0}$.

self as a broad peak in the conductivity spectra of CNT films and composites ${ }^{16,27,46}$. Based on the data on the LPR peak at $2.3 \mathrm{THz}$ in Fig. 6, we may conclude that (i) the magnitude of this peak does not depend on whether tubes are isolated or form a conductive network; (ii) the high frequency side of this peak practically does not depend on the intertube coupling (though, it depends on the electron relaxation time in the CNTs and on the type of the distribution function over the CNT length); (iii) the low-frequency side of the peak strongly depends on the intertube interaction. The latter leads to the peak broadening in the direction of the lower frequencies. Also, above conclusions (i) and (ii) justify application of the homogenization theory of non-interacting nanoparticles to describe the effective permittivity of CNT-based composites and films at and above LPR frequency. Thus, the Waterman-Truell formula adapted for CNT-based media $^{25}$ gives a good approximation for the estimation of the effective parameters of CNT-based media in the terahertz and infrared ranges.

It should be noted that the conductivity peak reported at $3 \mathrm{THz}$ for thin CNT films in Ref. 45 was associated with plasmonic excitations due to reflections of the plasmon wave at the CNT intersections. Here, we have shown that such a reflection is impossible. The reasons are (i) the very week electromagnetic interaction between CNTs (see Figs. 3 and 5), and (ii) negligibly small modification of the intrinsic electron transport in the CNT near the crossing point ${ }^{34,36}$.

Though the intertube conductance $G_{d}$ depends on the angle $\theta$ between the crossing tubes ${ }^{32,33}$, we shall not take this into account in our further consideration. Figures $9(\mathrm{a}, \mathrm{b})$ show the spectra of the imaginary part of the polarizability of the 1st CNT at different angles $\theta$ and different distances $r$; the intertube conductance is assumed to be the same $\left(G_{d}=0.1 G_{0}\right)$. As shown in Fig. 9 , the

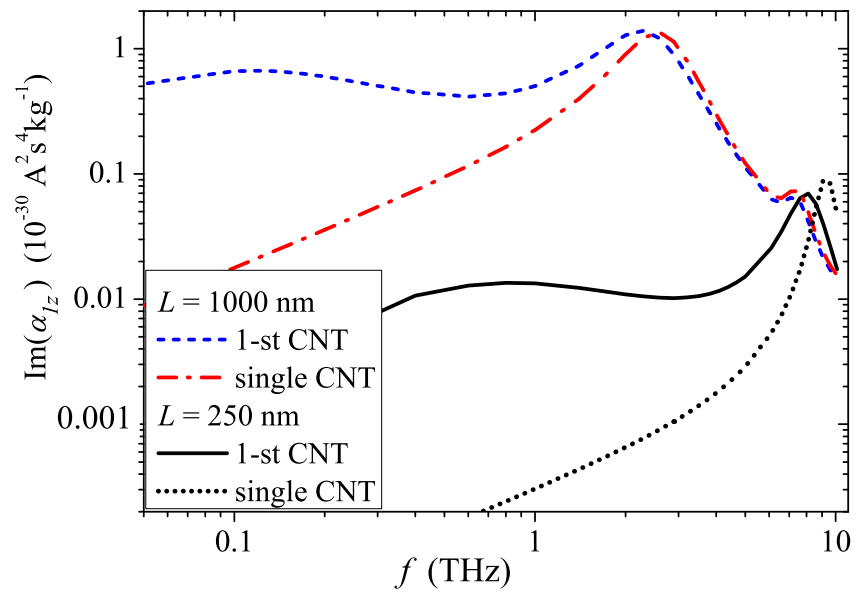

FIG. 10. Frequency dependence of the imaginary part of the polarizability of a single CNT and the 1st CNTs for various tube lengths, $L=250 \mathrm{~nm}$ and $L=1000 \mathrm{~nm} ; r=0.1 L$, $\theta=45^{\circ} ; G_{d}=0.1 G_{0}$.

angle between the tubes slightly affects the tube polarizability, whereas distance $r$ impacts significantly the value of $\operatorname{Im}\left(\alpha_{1 z}\right)$. Below the LPR peak, the energy dissipation in the CNTs is higher for smaller distance $r$. This occurs due to the following reasons: (i) the charge density is maximal near the CNT edges resulting in the highest intertube voltage and current; (ii) the total size of the CNT system is larger at smaller $r$ causing smaller depolarizing field and, consequently, the higher current density excited in the CNTs.

Figure 10 shows the spectra of the imaginary part of the polarizability of a single CNT and the 1st CNT for various tube lengths $L=250 \mathrm{~nm}$ and $L=1 \mu \mathrm{m}$ and constant values of $r / L=0.1, \theta$, and $G_{d}$. As shown in Fig. 10 , the polarizability peaks are blue shifted with decreasing tube lengths. The influence of the intertube coupling is strong in the quasi-static regime of the electromagnetic interaction, i.e. below the localized plasmon resonance. The intertube current and its impact depend not only on the conductivity of the intertube junction, but also on the intertube voltage. The voltage, in turn, is caused by depolarization effects and, consequently, it strongly depends on the frequency, tube length, and relative position of the intertube junction. Thus, the intertube coupling is determined by the finite-size effect in the CNTs.

\section{CONCLUSION}

Electromagnetic boundary-value problem of two crossing finite-length SWCNTs has been formulated taking into account the electromagnetic coupling and intertube charge transport. The problem has been reduced to Pocklington and Hallén-type equations with respect to the current density in CNTs and intertube current. The intertube current has been taken into account by (i) a 
discontinuity of the axial currents in the CNTs, and (ii) by an introduction of the extra charges at the crossing points of the CNTs. To incorporate the field of extra charges into the Hallen-type equation, they have been replaced by semi-infinite currents.

The current in the CNTs can be divided into two components; one of them describes the charge oscillations within the tubes, and the other - describes the oscillation of the charge passing between the tubes. The intertube current is shown to behave like the current in an equivalent RC-circuit. The cutoff frequency of the RC-circuit separates the regimes of high and low transparency of the intertube junction.

The electromagnetic interaction between the crossing tubes occurs due to the interaction of the charges concentrated near the crossing point; these charges create strong radial scattered field which can be hundreds fold higher than the incident field resulting in the field enhancement in the gap between the tubes. Electromagnetic interaction of the CNTs with zero intertube conductance shifts slightly the LPR peak to the lower or higher frequencies depending on the direction of the incident field. Due to the intertube current, the charge is redistributed between the tubes resulting in a weakening of the depolarizing field in the CNTs and in appearance of the depolarizing field in the gap between the CNTs. The intertube current leads to a significant energy dissipation near the crossing point of the CNTs at low frequencies resulting in manifold increase of the total energy dissipation. The intertube coupling practically does not change the magnitude and frequency of the LPR peak, but modifies its low-frequency side. Due to a weak intertube coupling at and above the LPR peak, the Waterman-Truell formula is a good approximation for the description of the effective permittivity of CNT-networks.

\section{ACKNOWLEDGMENTS}

This research was partially funded by the H2020MSCA-RISE project 823878 - TERASSE. IAS acknowledges support from the megagrant 14.Y26.31.0015 and Goszadanie no. 3.8884.2017/8.9 of the Ministry of Education and Science of Russian Federation. PK is supported by Horizon 2020 IF TURANDOT project 836816 and the Academy of Finland Flagship Programme, Photonics Research and Innovation (PREIN), decision 320166. S.A.M. and M.V.S. also are thankful for support by Tomsk State University Competitiveness Improvement Program.

\section{Appendix A: Application of the four-terminal Landauer formula to the junction of the crossing CNTs}

Junction between two crossing CNTs can be modeled as a four-terminal mesoscopic system. Each short sec-

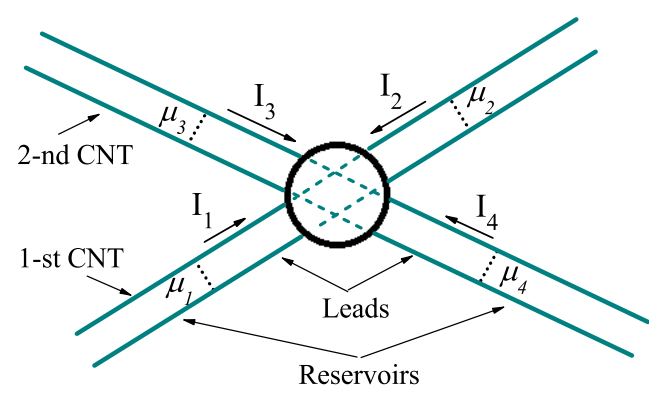

FIG. 11. Schematic illustration of two crossing CNTs decomposed following the Landauer-Büttiker concept of quantum transport. CNT junction (black circle) has four terminals connected by leads to reservoirs.

tion of the tubes near the junction plays a role of a lead connecting the junction to the rest of the tube which is considered as a reservoir (see Fig. 11). Let the electrochemical potentials of the $n$-reservoirs $(m, n=1,2 . .4)$ be $\mu_{n}$, and the current in the $n$-lead be $I_{n}$. The relation between $\mu_{n}$ and $I_{n}$ in the case of multichannel leads can be expressed by the four-terminal Landauer formula ${ }^{47,48}$ :

$$
I_{n}=\frac{e}{h}\left(\left(N_{n}-R_{n n}\right) \mu_{n}-\sum_{m \neq n} T_{n m} \mu_{m}\right) .
$$

where $N_{n}$ is a number of conductive channels in the $n$th lead, $N_{n}=2$ for metallic undoped SWCNT ${ }^{49}$. Also in A1, $T_{n m}$ is a total transmission function for carriers incident in the $m$ th lead to be scattered by junction into the lead $n ; R_{n n}$ is a total reflection function for carriers incident in the $n$th lead to be reflected into the the same lead, thus

$$
T_{n m}=\sum_{i=1}^{N_{n}} \sum_{j=1}^{N_{m}} T_{n m, i j}, \quad R_{n n}=\sum_{i=1}^{N_{n}} \sum_{j=1}^{N_{n}} R_{n n, i j},
$$

where $T_{n m, i j}$ is a probability for carriers incident in the channel $j$ of the $m$ th lead to be transmitted into the channel $i$ of the $n$th lead ; $R_{n n, i j}$ is a probability for carriers incident in the $j$ th channel of the $n$th lead to be reflected into the $i$ th channel of the same lead.

If the electrochemical potentials $\mu_{i}$ is the same in all reservoirs, the currents must vanish in the leads, i.e. $I_{n}=$ 0 . In this case, (A1) is transformed into the following expressions:

$$
R_{n n}+\sum_{m \neq n} T_{n m}=N_{n}, .
$$

Carrier transmission coefficients between two of the four terminals $T_{n m}$ can be found from ab initio quantummechanical calculations ${ }^{34,36,37}$.

Let us now determine the relation between $T_{n m}$ and two-terminal intertube conductance value $G_{d}$ used in 
formula (9). For simplicity, we consider the case when the external magnetic field is absent. Due to the timereversal invariance and the symmetry of the lead positions with respect to the junction, we can write the following relations for intertube transmission coefficients: $T_{13}=T_{31}=T_{24}=T_{42}$ and $T_{14}=T_{41}=T_{23}=T_{32}$, and for intratube transmission coefficients: $T_{12}=T_{21}$ and $T_{34}=T_{43}{ }^{47,48}$.

Let us next assume that four arbitrary electrochemical potentials $\mu_{n}$ are applied to four terminals of the junction. Using (A3) and relations between intratube and intertube transmission coefficients introduced above, we can rewrite equations (A1) in the following way:

$$
\left\{\begin{array}{l}
I_{1}=\frac{e}{h}\left(T_{12} \Delta \mu_{12}+T_{13} \Delta \mu_{13}+T_{14} \Delta \mu_{14}\right) \\
I_{2}=-\frac{e}{h}\left(T_{12} \Delta \mu_{12}-T_{23} \Delta \mu_{23}-T_{24} \Delta \mu_{24}\right) \\
I_{3}=\frac{e}{h}\left(T_{34} \Delta \mu_{34}+T_{31} \Delta \mu_{31}+T_{32} \Delta \mu_{32}\right) \\
I_{4}=-\frac{e}{h}\left(T_{34} \Delta \mu_{34}-T_{41} \Delta \mu_{41}-T_{42} \Delta \mu_{42}\right) .
\end{array}\right.
$$

where $\Delta \mu_{n m}=\mu_{n}-\mu_{m}, n, m=1,2 \ldots 4$. The first term in each equation of (A4) corresponds to the intratube transport, while other two terms are responsible for the intertube tunneling.

After adding the first equation to the second one and the third equation to the fourth one in (A4), we obtain:

$I_{t}=I_{1}+I_{2}=-\left(I_{3}+I_{4}\right)=\frac{e}{h}\left(T_{13}+T_{14}\right)\left(\Delta \mu_{13}+\Delta \mu_{24}\right)$,

where $I_{t}$ is the total intertube current.

As mentioned in section III, due to the depolarization effect, the total axial field in CNT is much lower than the radial field determining the intertube voltage. This means that for short length leads (about several nanometers) we can accept that $\mu_{3}=\mu_{4}, \mu_{1}=\mu_{2}$ and, consequently, $\Delta \mu_{13}=\Delta \mu_{24}=e U$, where $U$ is the intertube voltage. Then Eq. (A5) is transformed into (16) with the following intertube conductance

$$
G_{d}=\frac{2 e^{2}}{h}\left(T_{13}+T_{14}\right)
$$

\section{Appendix B: Pocklington and Hallén type equations for the 2nd CNT}

Following Sec. II B, Pocklington type equation can be formulated for the 2nd CNT in the coordinate system $\left(\rho_{2}, \phi_{2}, z_{2}\right)$

$$
\begin{gathered}
\frac{j_{2}\left(z_{2}\right)}{\sigma_{2}}=E_{2 z}^{(0)}(z)+E_{2 z}^{(1)}(z)+E_{2 z}^{(1 t)}(z)+ \\
\frac{i a_{2}}{\varepsilon \omega}\left(\int_{0}^{L-r_{2}-0}+\int_{L-r_{2}+0}^{L_{2}} \frac{\partial j_{2}\left(z^{\prime}\right)}{\partial z^{\prime}} \frac{\partial G\left(a_{2}, z_{2}-z^{\prime}\right)}{\partial z} d z^{\prime}\right)+ \\
\frac{i a_{2}}{\varepsilon \omega} \int_{0}^{L_{2}} k^{2} j_{2}\left(z^{\prime}\right) G\left(a_{2}, z_{2}-z^{\prime}\right) d z^{\prime},
\end{gathered}
$$

where $E_{2 z}^{(1)}$ and $E_{2 z}^{(1 t)}$ are axial components of an electric field produced by the current $j_{1}$ and charge $+q_{t}$, respectively, on the surface of the 2nd CNT.

Following Secs. II A and II C, Hallén type equation can be formulated for the 2 nd CNT in the coordinate system $\left(\rho_{2}, \phi_{2}, z_{2}\right)$

$$
\begin{gathered}
\int_{0}^{L_{2}}\left[\frac{1}{\sigma_{2}} \mathrm{e}^{i k\left|z_{2}-z_{2}^{\prime}\right|}+\frac{2 a_{2} k}{\varepsilon \omega} G\left(a_{2}, z_{2}-z_{2}^{\prime}\right)\right] j_{2}\left(z_{2}^{\prime}\right) d z_{2}^{\prime}+ \\
\frac{k I_{t}}{\pi \varepsilon \omega} \int_{L_{2}-r_{2}}^{\infty} G\left(a_{2}, z_{2}-z_{2}^{\prime}\right) d z_{2}^{\prime}=\Phi_{2}\left(z_{2}\right),
\end{gathered}
$$

where

$$
\begin{gathered}
\Phi_{2}\left(z_{2}\right)=\int_{0}^{L_{2}}\left(E_{2 z}^{(0)}\left(z_{2}\right)+\tilde{E}_{2 z}^{(1)}\left(z_{2}\right)\right) \mathrm{e}^{i k\left|z_{2}-z_{2}^{\prime}\right|} d z_{2}^{\prime}+ \\
\left\{\begin{array}{l}
C^{\prime}{ }_{1} \mathrm{e}^{i k z_{2}}+C_{2}^{\prime} \mathrm{e}^{-i k z_{2}}, z_{2} \in\left(0, L_{2}-r_{2}\right) \\
D_{1}^{\prime} \mathrm{e}^{i k z_{2}}+D_{2}^{\prime} \mathrm{e}^{-i k z_{2}}, z_{2} \in\left(L_{2}-r_{2}, L_{2}\right)
\end{array}\right.
\end{gathered}
$$

Here $C_{1,2}^{\prime}$ and $D_{1,2}^{\prime}$ are unknown constants to be determined from the edge conditions (5)-(7); and $\tilde{E}_{2 z}^{(1)}$ is an axial field produced by the currents $j_{1}$ and $j_{1 a}$ on the surface of the 2nd CNT.

\section{Appendix C: Electric field potential of the semi-infinite current}

Let us consider the axial current on the surface of infinite-length cylinders which extending the 1st CNT

$$
j_{1 a}(z)=\frac{1}{2 \pi a_{1}}\left\{\begin{array}{l}
I_{1}, z \in\left(-\infty, r_{1}\right) \\
I_{2}, z \in\left(r_{1},+\infty\right)
\end{array},\right.
$$

where $I_{1,2}$ are constant values. Herzt potential of the field produced by the current $j_{1 a}$ can be expressed as follows

$$
\Pi_{t}(\rho, z)=\frac{i}{2 \pi \varepsilon \omega} \int_{-\infty}^{\infty} j_{1 a}(z) G\left(\rho, z-z^{\prime}\right) d z^{\prime},
$$

where $G\left(\rho, z-z^{\prime}\right)$ is a Green function defined by (18). The electric potential at point $(\rho, z)$ can be calculated as

$$
\begin{aligned}
\varphi_{t}=-\frac{\partial \Pi_{t}(\rho, z)}{\partial z}= & -\frac{i}{2 \pi \varepsilon \omega}\left[I_{1} \int_{-\infty}^{r_{1}} \frac{\partial G\left(\rho, z-z^{\prime}\right)}{\partial z} d z^{\prime}+\right. \\
& \left.I_{2} \int_{r_{1}}^{\infty} \frac{\partial G\left(\rho, z-z^{\prime}\right)}{\partial z} d z^{\prime}\right] . \quad \text { (C3) }
\end{aligned}
$$

Due to the symmetry of the integrals in (C3) with respect to the replacement $\partial / \partial z \rightarrow-\partial / \partial z^{\prime}$, the electric potential can be calculated as

$$
\varphi_{t}(\rho, z)=\frac{i\left(I_{1}-I_{2}\right)}{2 \pi \varepsilon \omega} G\left(\rho, z-r_{1}\right)
$$

The value of $\varphi_{t}$ in $(\mathrm{C} 4)$ coincides with an electrical potential of the field produced by the charge $q_{t}=i I_{t} / \omega=$ 
$i\left(I_{1}-I_{2}\right) / \omega$ distributed uniformly on an infinitely thin ring of a radius $a_{1}$ at $z=r_{1}$. The difference between the $z$-component of the electric field $\partial^{2} \Pi_{t} / \partial z^{2}+k^{2} \Pi_{t}$ produced by the current $j_{1 a}$ and the $z$-component of the field $-\partial \varphi_{t} / \partial z$ produced by the charge $+q_{t}$ equals $k^{2} \Pi_{t}$, and it can be neglected on the surfaces of the CNTs when $\left(k L_{1,2}\right)^{2} \ll 1$. Then, the electric field produced by the charge $+q_{t}$ can be replaced by the field produced by the currents $j_{1 a}$. This replacement is justified if the field produced by the currents $j_{1 a}$ on the CNT surface can be considered longitudinal with a high accuracy. Though the choice of the values $I_{1,2}$ is limited only by an equality $I_{1}-I_{2}=I_{t}$, it is reasonable to choose them to be close to zero in order to guarantee the longitudinal character of the field $\Pi_{t}$ in the vicinity of the CNTs. For simplicity we choose $I_{1}=I_{t}$ and $I_{2}=0$ for $j_{1 a}$ in (24). Thus, the electric field produced by the charges $+q_{t}$ and $q_{t}$ in the CNTs can be replaced by the field produced by the currents $j_{1 a}$ and $j_{2 a}$, respectively, as shown in Fig. $2(b, c)$.
${ }^{1}$ G. Y. Slepyan, S. A. Maksimenko, A. Lakhtakia, O. Yevtushenko, and A. V. Gusakov, Phys. Rev. B 60, 17136 (1999).

2 P. J. Burke, S. Li, and Z. Yu, IEEE Trans. Nanotechnol. 5, 314 (2006).

${ }^{3}$ G. W. Hanson, IEEE Trans. Antennas Propag. 53, 3426 (2005).

${ }^{4}$ G. Y. Slepyan, M. V. Shuba, S. A. Maksimenko, and A. Lakhtakia, Phys. Rev. B 73, 195416 (2006).

${ }^{5}$ R. R. Hartmann, J. Kono, and M. E. Portnoi, Nanotechnology 25, 322001 (2014).

${ }^{6}$ M. V. Shuba, A. G. Paddubskaya, A. O. Plyushch, P. P. Kuzhir, G. Y. Slepyan, S. A. Maksimenko, V. K. Ksenevich, P. Buka, D. Seliuta, I. Kasalynas, J. Macutkevic, G. Valusis, C. Thomsen, and A. Lakhtakia, Phys. Rev. B 85, 165435 (2012).

7 M. V. Shuba, A. V. Melnikov, A. G. Paddubskaya, P. P. Kuzhir, S. A. Maksimenko, and C. Thomsen, Phys. Rev. B 88, 045436 (2013).

${ }^{8}$ M. V. Shuba, A. V. Melnikov, P. P. Kuzhir, S. A. Maksimenko, G. Y. Slepyan, A. Boag, A. M. Conte, O. Pulci, S. Bellucci, Phys. Rev. B 96, 205414 (2017).

${ }^{9}$ M. V. Shuba, S. A. Maksimenko, and A. Lakhtakia, Phys. Rev. B 76, 155407 (2007).

10 M. V. Shuba, G. Y. Slepyan, S. A. Maksimenko, C. Thomsen, and A. Lakhtakia, Phys. Rev. B 79, 155403 (2009).

11 A. M. Hassan, F. Vargas-Lara, J. F. Douglas, E. J. Garboczi, IEEE T. Antenn. Propag. 642743 (2016).

12 M. V. Shuba, G. Y. Slepyan, S. A. Maksimenko, and G. W. Hanson, J. Appl. Phys. 108, 114302 (2010).

13 M. V. Shuba, A. G. Paddubskaya, P. P. Kuzhir, S. A. Maksimenko, G. Valusis, M. Ivanov, J. Banys, V. Ksenevich, G. W. Hanson, Mater. Res. Express 4, 075033 (2017).

14 I. V. Bondarev, G. Y. Slepyan, S. A. Maksimenko, Phys. Rev. Lett. 89, 115504 (2002).

15 A. M. Nemilentsau, G. Ya. Slepyan, and S. A. Maksimenko, Phys. Rev. Lett. 99, 147403 (2007).

16 A. Ugawa, A. G. Rinzler, and D. B. Tanner, Phys. Rev. B 60, R11305 (1999).

17 M.V. Shuba, A. Paddubskaya, P.P. Kuzhir, S.M. Maksimenko, E. Flahaut, V. Fierro, A. Celzard, G. Valusis, J. Phys. D. Appl. Phys. 50, 08LT01 (2017).

18 J. Hao, and G. W. Hanson, Phys. Rev. B 74, 035119 (2006).

19 I. S. Nefedov, Phys. Rev. B 82, 155423 (2010).

20 S. Mikki, A. Kishk, Prog. Electromagnet. Res. C 83, 83 (2018).

${ }^{21}$ G. Slepyan, A. Boag, V. Mordachev, E. Sinkevich, S. Maksimenko, P. Kuzhir, G. Miano, M. E. Portnoi, and A. Maf- fucci, New J. Phys. 19, 023014 (2017).

${ }^{22}$ G. Ya. Slepyan, A. Boag, V. Mordachev, E. Sinkevich, S. Maksimenko, P. Kuzhir, G. Miano, M. E. Portnoi, and A. Maffucci, IEEE Trans. Ellectromagn. Compat. 57, 1645 (2015).

23 C. Forestiere, A. Maffucci, S. A. Maksimenko, G. Miano, and G. Ya. Slepyan, IEEE T. Nanotechnol. 11554 (2012).

24 A.I. Luchaninov, D.V. Gretskih, E.A. Medvedev, A.S. Chemerovskyi, Telecommunications and Radio Engineering 74, 1327 (2015).

25 G. Ya. Slepyan, M. V. Shuba, S. A. Maksimenko, C. Thomsen, and A. Lakhtakia, Phys. Rev. B 81, 205423 (2010).

${ }^{26}$ M. V. Shuba, D. I. Yuko, P. P. Kuzhir, S. A. Maksimenko, M. A. Kanygin, A. V. Okotrub, R. Tenne, Ph. Lambine, Carbon 129, 688 (2018).

27 P. Karlsen, M. V. Shuba, C. Beckerleg, D. I. Yuko, P. P. Kuzhir, S. A. Maksimenko, V. Ksenevich, Ho Viet, A. G. Nasibulin, R. Tenne, and E. Hendry, J. Phys. D: Appl. Phys. 51, 014003 (2018).

${ }^{28}$ M. V. Shuba, D. I. Yuko, G. Gorokhov, D. Meisak, D.S. Bychanok, P. P. Kuzhir, S. A. Maksimenko, P. Angelova, E. Ivanov, and R. Kotsilkova, Mater. Res. Express 6, 095050 (2019).

29 Y.-G. Yoon, M. S. C. Mazzoni, H. J. Choi, J. Ihm, and S. G. Louie, Phys. Rev. Lett. 86, 688 (2001).

30 S. Tasaki, K. Maekawa, and T. Yamabe, Phys. Rev. B 57, 9301 (1998).

31 M. S. Fuhrer, J. Nygard, L. Shih, M. Forero, Y.-G. Yoon, M. S. C. Mazzoni, H. J. Choi, J. Ihm, S. G. Louie, A. Zettl, P. L. McEuen, Science 288, 494 (2000).

32 A. Buldum and J. P. Lu, Phys. Rev. B 63, 161403(R) (2001).

33 A. A. Maarouf and E. J. Mele, Phys. Rev. B 83, 045402 (2011)

34 P. Havu, M. J. Hashemi, M. Kaukonen, E. T. Seppala, and R. M. Nieminen, J. Phys.: Condens. Matter 23, 112203 (2011).

35 S. Dag, R. T. Senger, and S. Ciraci, Phys. Rev. B 70, 205407 (2004).

36 D. J. Mowbray, C. Morgan, and K. S. Thygesen, Phys. Rev. B 79, 195431 (2009).

37 T. Ketolainen, V. Havu, and M. J. Puska, J. Chem. Phys. 142, 054705 (2015).

${ }^{38}$ L. A. Weinstein, The Theory of Diffraction and the Factorization Method (Golem, New York, 1969).

39 E. Forati, A. D. Mueller, P. G. Yarandi, and G. W. Hanson, IEEE Trans. Antennas Propag. 59, 4355 (2011).

40 V. L. Gurevich, Phys. Rev. B 55, 4522 (1997). 
41 P. Nordlander, C. Oubre, E. Prodan, K. Li, and M. I. Stockman, Nano Lett. 4, 899 (2004).

${ }^{42}$ P. K. Jain, S. Eustis, and M. A. El-Sayed, J. Phys. Chem. B 110, 18243 (2006).

${ }^{43}$ K. D. Osberg, N. Harris, T. Ozel, J. C. Ku, G. C. Schatz, C. A. Mirkin, Nano Lett. 14 (12), 6949 (2014).

44 T.-S. Deng, J. Parker, Y. Yifat, N. Shepherd, N. F. Scherer, J. Phys. Chem. C 122 (48), 27662 (2018).

45 E. S. Zhukova, A. K. Grebenko, A. V. Bubis , A. S. Prokhorov, M. A. Belyanchikov, A. P. Tsapenko, E. P.
Gilshteyn, D. S. Kopylova, Yu. G. Gladush, A. S. Anisimov, V. B. Anzin, A. G. Nasibulin and B. P. Gorshunov, Nanotechnology 28, 445204 (2017).

46 Q. Zhang, E. H. Haroz, Z. Jin, L. Ren, X. Wang, R. S. Arvidson , A. Luttge, and J. Kono, Nano Lett. 13, 5991 (2013).

47 M. Büttiker, Phys. Rev. Lett. 57, 1761 (1986).

48 M. Büttiker, IBM J. Res. Dev. 32, 317 (1988).

49 H.-S. Sim, C.-J. Park, and K. J. Chang, Phys. Rev. B 63, 073402 (2001). 